\title{
DELETED PRODUCTS WITH PRESCRIBED HOMOTOPY TYPES
}

ARTHUR H. COPELAND, JR. ${ }^{1}$

1. Introduction. This work is concerned with the problem of deciding when a space $Y$ has the homotopy type of a deleted product $R X=X \times X$-diagonal. That is, given $Y$ find spaces $X$ such that $R X$ has the homotopy type of $Y . \S 2$ argues that this is a reasonable program by briefly reviewing a small segment of embedding theory. The next two sections present techniques useful in solving the problem, and also note that there are spaces $Y$ not having the homotopy type of any deleted product. The last two sections apply these techniques to obtain some solutions for the cases $Y=S^{1}$ and $Y=S^{2}$.

I wish to thank M. E. Mahowald and R. F. Williams for many valuable suggestions.

2. Obstructions to embeddings. Let $X$ and $Y$ be Hausdorff spaces. On the deleted product

$$
R X=\left\{\left(x, x^{\prime}\right) \mid x, x^{\prime} \in X \text { and } x \neq x^{\prime}\right\}
$$

there is the involution $T_{X}\left(x, x^{\prime}\right)=\left(x^{\prime}, x\right)$. Let $R_{*} X=R X / T_{X}$ and $E(X, Y)=(R X \times R Y) /\left(T_{X} \times T_{Y}\right)$ be orbit spaces, and let $\pi: E(X, Y) \rightarrow R_{*} X$ be the map induced by the projection $R X \times R Y$ $\rightarrow R X$. Using the fact that $X$ is Hausdorff, one easily verifies that $\pi$ is the projection map of a fiber bundle with fiber $R Y$ and group $Z_{2}$ (= the integers modulo 2).

An embedding $f: X \rightarrow Y$ induces a $Z_{2}$-equivariant map $R f: R X \rightarrow R Y$, and hence an equivariant map

$$
R X \stackrel{\text { diagonal }}{\longrightarrow} R X \times R X \stackrel{1 \times R f}{\longrightarrow} R X \times R Y .
$$

It is immediate that the induced map $R_{*} X \rightarrow E(X, Y)$ is a cross section for $\pi$. Thus if $B_{n}$ is the standard bundle of coefficients $\pi_{n}(R Y)$ over $R Y$, the vanishing of the obstructions

$$
\gamma^{n+1}(\pi) \in B^{n+1}\left(R_{*} X ; B_{n}\right)
$$

for all $n$ is a necessary condition for the existence of an embedding. These ideas have been studied by van Kampen [6], Whitney [8], Wu [9], Shapiro [5], Haefliger [3] and Weber [7].

Received by the editors May 10, 1967.

1 This research was partially supported by NSF Grant GP 5591 
The moral I wish to draw from this is simply that in studying embeddings $X \rightarrow Y$, the homotopy types of $R X$ and $R Y$ are of fundamental importance. Since obstruction theory plays a role here one needs to build universal examples for the obstructions. It is a reasonable conjecture that these examples would be more valuable if they were deleted products themselves. Thus one is confronted with the problem: given a space $P$, find $X$ such that $R X$ has the homotopy type of $P$.

3. Euler-Poincare characteristics. Suppose $T$ is a finite triangulation of a space $X$. Wu [9] shows that

$$
J T=\bigcup\{\sigma \times \tau \mid \sigma, \tau \in T \text { and } \sigma \cap \tau=\varnothing\}
$$

is a deformation retract of $R X$ (the simplexes $\sigma, \tau$ are assumed to be closed). Thus the Euler-Poincare characteristic $\chi R X$ is the same as that of $J T$. Since the number of cells $\sigma \times \tau$ in $J T$ is even, one immediately makes the following observation.

(3.1) $\chi R X$ is an even integer. Thus there exist spaces which do not have the homotopy type of any deleted product.

Let $T_{p}$ be the set of $p$-simplexes in the triangulation $T$. If $\sigma, \tau \in T$, let $\langle\sigma, \tau\rangle=0$ when $\sigma \cap \tau=\varnothing$ and $\langle\sigma, \tau\rangle=1$ otherwise. Since a cell $\sigma \times \tau$ appears in $J T$ if and only if $\langle\sigma, \tau\rangle=0$,

$$
\begin{aligned}
\chi J T & =\sum\left\{(-1)^{p+q}(1-\langle\sigma, \tau\rangle) \mid \sigma \in T_{p},\right. \\
& \left.\quad \tau \in T_{q} ; p, q=0,1,2, \cdots\right\} \\
& =(\chi X)^{2}-\sum(-1)^{p+q}\langle\sigma, \tau\rangle .
\end{aligned}
$$

Now suppose $\operatorname{dim} X=1$. Let $c_{k}$ be the number of simplexes in $T_{k}$. If $\sigma \in T_{0}$, the order of $\sigma$ is

$$
O(\sigma)=\sum\left\{\langle\sigma, \tau\rangle \mid \tau \in T_{1}\right\} .
$$

Let $p_{n}=p_{n}(X)$ be the number of 0 -simplexes of order $n$, and abbreviate $\chi=\chi X$. Then we have

$$
\begin{gathered}
\sum\left\{\langle\sigma, \tau\rangle \mid \sigma, \tau \in T_{0}\right\}=c_{0}=\sum p_{n}, \\
\sum\left\{\langle\sigma, \tau\rangle \mid \sigma \in T_{0}, \tau \in T_{1}\right\}=\sum_{\sigma}\left(\sum_{\tau}\langle\sigma, \tau\rangle\right)=\sum n p_{n}, \\
\sum\left\{\langle\sigma, \tau\rangle \mid \sigma, \tau \in T_{1}\right\} \\
=c_{1}+\sum\left\{\langle\sigma, p\rangle\langle p, \tau\rangle \mid p \in T_{0}, \sigma \neq \tau\right\} \\
=c_{1}+\sum_{p}\left(\sum_{\sigma}\langle p, \sigma\rangle\left(\sum_{\tau \neq \sigma}\langle p, \tau\rangle\right)\right) \\
=c_{1}+\sum_{n(n-1) p_{n} .} n
\end{gathered}
$$


Using (3.2), (3.3) and the fact that $\chi=c_{0}-c_{1}$, we get

$$
\chi R X=\chi^{2}+\chi-\sum(n-2)(n-1) p_{n} .
$$

This yields the topological invariant,

$$
\lambda=\lambda(X)=\sum(n-2)(n-1) p_{n},
$$

which will be used in the last two sections. Variants of (3.4) may be obtained by noting that

$$
\sum n p_{n}=\sum_{\tau} \sum_{\sigma}\langle\sigma, \tau\rangle=2 c_{1}
$$

$\left(\sigma \in T_{0}\right.$ and $\left.\tau \in T_{1}\right)$, whence $2 \chi=\sum(2-n) p_{n}$.

4. Suspensions. Define the suspension of a space $X$ to be the join,

$$
S X=X * 2 \text {, }
$$

where $2=\{a, b\}$ is a discrete space with exactly two points.

(4.1) ThEOREM. If $X$ is finitely triangulable, then RSX has the homotopy type of $S R X$.

Proor. Let $T$ be a triangulation of $X$ and let $S T$ be the induced triangulation of $S X$. Set

$$
\begin{aligned}
& X_{1}=U\{(\sigma * a) \times(\tau * b) \mid \sigma, \tau \in T, \sigma \cap \tau=\varnothing\}, \\
& X_{2}=U\{(\sigma * b) \times(\tau * a) \mid \sigma, \tau \in T, \sigma \cap \tau=\varnothing\} .
\end{aligned}
$$

It is easily checked that $J S T=X_{1} \cup X_{2}$ and $J T=X_{1} \cap X_{2}$. The theorem now follows from the observation that $X_{1}$ and $X_{2}$ are contractible.

The author claims no priority here. This result seems to be known but unpublished.

Note that in this theorem one must use the classical suspension, rather than the reduced suspension. For example, if $X=k$ is a discrete space with $k$ points, then $\chi R S X=\chi S R X=2+k-k^{2}$. But the reduced suspension $S^{\prime} X$ is a wedge of $k-1$ circles. It follows from (3.4) that $\chi R S^{\prime} X=-3(k-1)(k-2)$.

\section{Homotopy 1-spheres.}

(5.1) Theorem. Suppose $X$ is a 1-dimensional, finitely triangulable space. Then $R X$ is a homotopy 1-sphere iff

(a) $X$ has no pair of disjoint simple closed curves,

(b) $X$ is connected, and

(c) $\lambda(X)=\chi^{2}+\chi$.

Proof. If $X_{1}$ and $X_{2}$ are disjoint closed subsets of $X=X_{1} \cup X_{2}$, then $R X=R X_{1} \cup R X_{2} \cup X_{1} \times X_{2} \cup X_{2} \times X_{1}$. Furthermore, the only con- 
nected finitely triangulable space $Y$ with $R Y$ disconnected is an arc [4]. Thus $R X$ is connected iff $X$ is connected and not an arc. If $X$ contains a pair of disjoint simple closed curves $C_{1}, C_{2}$, then $C_{1} \times C_{2}$ and $C_{2} \times C_{1} \subset R X$ carry nontrivial 2-dimensional homology classes.

Now suppose $R X$ is a homotopy 1 -sphere. We have just seen that (a) and (b) must hold. Furthermore, $\chi R X=\chi\left(S^{1}\right)=0$, whence (c) follows from (3.4).

Conversely, suppose (a), (b) and (c) hold. Condition (a) implies that the Betti number $\beta_{2}(R X)=0$ and condition (b) implies $\beta_{0}(R X)$ $=1$ (with (c) used to eliminate the case $X=$ an arc). Condition (c) and (3.4) imply $\chi R X=0$, whence $\beta_{1}(R X)=1$. C. W. Patty shows when $X$ is 1 -dimensional and finitely triangulable, $H_{*}(R X)$ is free abelian and $\pi_{1}(R X, *)$ is a freely generated group. Thus $R X$ is a homotopy 1-sphere.

It is well known that if $X$ is a 1 -sphere or a triod, then $R X$ is a homotopy 1-sphere. I conjecture that there are no other 1-dimensional spaces with this property.

AdDED IN Proof. C. W. Patty has pointed out to me that condition (a) in Theorems (5.1) and (6.1) is insufficient to insure that $R X$ have no 2-cycles. The houses-and-wells configuration and the complete graph on five vertices are counterexamples. In each theorem, the condition should read

(a) $X$ may be embedded in the plane, and contains no pair of disjoint simple closed curves.

A proof of this should appear soon.

6. Homotopy 2-spheres. According to Theorem 4.1, any suspension of one of the spaces constructed in $\$ 5$ will have its deleted product be a homotopy 2-sphere. Only one of these is contractible. In this section we construct cones $C X$, with $X$ a 1-dimensional space, such that $R C X$ is a homotopy 2 -sphere.

(6.1) Theorem. Suppose $X$ is a 1-dimensional, finitely triangulable space. Then $R C X$ is a homotopy 2-sphere iff

(a) $X$ has no pair of disjoint simple closed curves,

(b) $X$ is connected, and

(c) $\lambda=\chi^{2}-\chi+2$.

The proof of Theorem 6.1 is preceded by two lemmas which seem to be of independent interest.

(6.2) Lemma. If $X$ is a finitely triangulable connected space, other than a circle or an arc, then the fundamental group of $R C X$ is trivial.

Proof. Select a triangulation $T$ for $X$ and use the induced triangu- 
$C T=T * v$ of $C X=X * v$, where $v$ is some point not in $X$. It suffices to show that $\pi_{1}(J C T)$ is trivial. But $J C T=X_{1} \cup X_{2}$, where

$$
\begin{aligned}
& X_{1}=U\{(\sigma * v) \times \tau \mid \sigma \cap \tau=\varnothing\}, \\
& X_{2}=U\{\sigma \times(\tau * v) \mid \sigma \cap \tau=\varnothing\} .
\end{aligned}
$$

Since $X_{1} \cap X_{2}=J T$ is connected, it suffices to show that every simple closed curve in $X_{i}(i=1,2)$ bounds a disc in JCT. Since $v \times X \subset X_{1}$ and $X \times v \subset X_{2}$ are deformation retracts, it suffices to do this for curves $v \times C$ and $C \times v$ with $C$ a simple closed curve in $X$. Since $X$ is not a 1 -sphere, there is a point $p \in X-C$. Then $(p * v)$ $\times C \cup p \times(C * v)$ is a disc whose boundary is $v \times C$, and $C \times v$ is the boundary of $C \times(p * v) \cup(C * v) \times p$.

(6.3) Lemma. If $X$ is a finitely triangulable 1-dimensional space, then $\chi R C X=\lambda-\chi^{2}+\chi(\lambda=\lambda(X)$ and $\chi=\chi(X))$.

Proof. In the notation of Lemma 6.2, JCT $=X_{1} \cup X_{2}$ and $J T$ $=X_{1} \cap X_{2}$. Thus $\chi J C T+\chi J T=\chi X_{1}+\chi X_{2}=2 \chi$. The result now follows at once from (3.4).

Proof of Theorem (6.1). If $\beta_{k}(Y)$ is the $k$ th Betti number of a space $Y$, then

$$
\beta_{k+1}(R C X)-\beta_{k}(R X)+2 \beta_{k}(X)=0 \quad \text { for } k>0 ;
$$

$$
\beta_{1}(R C X)-\beta_{0}(R X)+2 \beta_{0}(X)-1=0,
$$

provided $X$ is not a 1-point space, a 2-point space or a 1-sphere [1]. Also, $R C X$ is connected.

Suppose (a), (b) and (c) are satisfied. Then $X$ is not a 1-point space, a 2-point space, an arc or a 1-sphere. The fact that $\operatorname{dim} X=1$, together with (6.4) insures that $\beta_{n}(R C X)=0$ for $n>3$. Condition (a) implies $\beta_{2}(R X)=0$, whence $\beta_{3}(R C X)=0$. Condition (b) and the remarks above imply $\beta_{0}(R X)=\beta_{0}(X)=1$, whence $\beta_{1}(R C X)=0$. Thus condition (c) and Lemma (6.3) imply $\beta_{2}(R C X)=1$. That is, $R C X$ is a homology 2-sphere. Lemma (6.2) now shows that $R C X$ is a homotopy 2 -sphere.

The proof of the converse follows obvious lines.

The triod, the $\theta$-curve and a space in the shape of the letter $P$ (without serifs) all have cones of the same isotopy type, and the deleted product of any one of these is a homotopy 2-sphere. Apart from these, all the spaces satisfying (a), (b), (c) and having distinct topological types have cones of distinct isotopy types [2]. A family 
of such spaces is obtained by taking $n$-gons and joining their vertices to their centers.

\section{REFERENCES}

1. A. H. Copeland, Jr., Deleted products of cones, Proc. Amer. Math. Soc. 16 (1965), 496-502.

2. - Isotopy of 2-dimensional cones, Canad. J. Math. 18 (1966), 201-210.

3. A. Haefliger, Plongement differentiables de variêtés dans variétés, Comment. Math. Helv. 36 (1961), 47-82.

4. C. W. Patty, The fundamental group of certain deleted product spaces, Trans. Amer. Math. Soc. 105 (1962), 314-321.

5. A. Shapiro, Obstructions to the imbedding of a complex in a euclidean space. The first obstruction, Ann. of Math. 66 (1957), 256-269.

6. E. R. Van Kampen, Komplexe in euklidischen Raumen, Abh. Math. Sem. Univ. Hamburg 9 (1932), 72-78.

7. C. Weber, Plongements de polyédres dans le domaine metastable, Comment. Math. Helv. 47 (1967), 1-27.

8. H. Whitney, The self-intersections of a smooth n-manifold in $2 n$-space, Ann. of Math. 45 (1944), 220-246.

9. W.-T. Wu, On the realisation of complexes in euclidean space, Sci. Sinica 7 (1958), 251-297, 365-387 and 8 (1959), 133-150.

NorthWESTERN UNIVERSITY 